\title{
ASCO 2019 - What's New in Thyroid Oncology?
}

\section{Jochen H. Lorch}

Department of Medical Oncology, Dana-Farber Cancer Institute, Harvard Medical School, Boston, MA, U.S.A. Member, International Oncology Thyroid Group (ITOG)

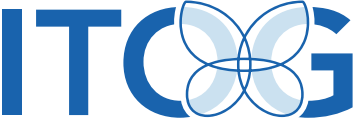

INTERNATIONAL THYROID ONCOLOGY GROUP
This editorial marks the introduction of a new, exciting collaboration between the American Thyroid Association (ATA) and the International Thyroid Oncology Group (ITOG). Several times a year, Dr. Jochen Lorch, a medical oncologist and member of both the ATA and ITOG, will review important topics in the thyroid cancer literature from this unique perspective. His first editorial here is timely and provides a summary of the most impactful scientific research presented at the recent annual meeting of the American Society of Clinical Oncology (ASCO) in Chicago (May 31-June 4, 2019).

The last days of May and the beginning of June: Time for barbecues, warm weather, and the pilgrimage to ASCO, the annual convention of the American Society of Clinical Oncology in Chicago, where over 40,000 people gather every year to discuss the latest developments in the field. This year's motto: "Caring for Every Patient, Learning From Every Patient" encapsulates challenges and opportunities rather well, given the current environment characterized by increasing health care costs and numerous discussions related to health care coverage.

Thyroid cancer research is traditionally included in the Head and Neck Cancer program and, as in past years, there were several remarkable abstracts presented at this year's ASCO meeting.

Two abstracts that were presented at Saturday's poster discussion session were centered around medullary and RET-driven thyroid cancers.
Abstract 6018: Matthew H. Taylor, Justin F. Gainor, Mimi I-Nan Hu, Viola Weijia Zhu, Gilberto Lopes, Sophie Leboulleux, Marcia S. Brose, Martin H. Schuler, Daniel W. Bowles, Dong-Wan Kim, Christina S Baik, Elena Garralda, Chia-Chi Lin, Douglas Adkins, Debashis Sarker, Giuseppe Curigliano, Hui Zhang, Corinne Clifford, Christopher D. Turner, Vivek Subbiah 2019 Activity and tolerability of BLU-667, a highly potent and selective RET inhibitor, in patients with advanced RET-altered thyroid cancers. J Clin Oncol 37(15 Suppl):6018. abstract. (ClinicalTrials. gov number NCT03037385.)

The abstract presented by Dr. Matthew Taylor and colleagues provided a preliminary update of an ongoing study with a RET inhibitor, BLU-667. BLU-667 is a novel, potent, and selective tyrosine kinase inhibitor (TKI), administered orally, that targets the RET proto-oncogene. RET is the principal driver in medullary thyroid cancer through point mutations within either the molecule's kinase domain or the extracellular domain. Approximately $20 \%$ of papillary thyroid cancer (PTC) cases are also driven by RET, typically through genetic fusion leading to dimerization and thus abnormal activation of RET signaling. This preliminary analysis provided results of the first 48 of 64 cases enrolled to date. Most patients had previously undergone treatment with another TKI and had documented disease progression or intolerance to standard of care with TKI treatment, such as cabozantinib or vandetanib, prior to enrollment. Results showed impressive radiographic response rates (53\% and 64\%, respectively) for patients with and without prior exposure to TKI. Also taking the rate of disease stability into account, the disease control rate (cases with complete response + partial response + stable disease) was an outstanding $94 \%$ 
and $97 \%$, respectively. Most importantly, safety and tolerability was favorable, at least as compared with standard anti-VEGF TKI therapy. Nonetheless, grade 3 hypertension was recorded in $23 \%$ of cases, with $16 \%$ attributed to treatment due to comparatively mild but present effects on VEGF receptors 1 and 2. Given the high efficacy and apparently favorable safety and tolerability, it is likely that BLU-667 will emerge as a new treatment standard in medullary thyroid cancer. It has recently been granted breakthrough status with the U.S. Food and Drug Administration (FDA) and will likely receive approval within the next year or so. Most likely, the main competitors will not be traditional TKIs such as cabozantinib and vandetanib, but rather another, similar RET-specific TKI that is also on track for FDA approval, Loxo-292. Data about Loxo-292 in MTC and RET-altered PTC presented at ASCO last year and updated at last year's ATA meeting paint a remarkably similar picture, with high response rates and manageable toxicity. Since there will likely be cross-resistance between BLU-667 and Loxo-292, the challenge will be which one to choose.

Abstract 6019: Dapeng Li, Ping Zhang Tang, Xiaohong Chen, Minghua Ge, Yuan Zhang, Zhuming Guo, Jun Wang, Feng Shi, Jiewu Zhang, Ying Cheng, Zhendong Li, Hui Liu, Jianwu Qin, Rui Huang, Ruochuan Cheng, Zhengang Xu, Yihebali Chi, Xiangqian Zheng, Ming Gao 2019 Anlotinib treatment in locally advanced or metastatic medullary thyroid carcinoma: a multicenter, randomized, double-blind, placebo-controlled phase IIB trial. J Clin Oncol 37(15 Suppl):6109. abstract. (ClinicalTrials.gov number NCT02586350.)

The second abstract presented at the Saturday poster session was submitted by Dr. Dapeng Li and colleagues from the Tianjin Medical University Cancer Institute in Tianjin, China. This was a multicenter, randomized, phase $2 \mathrm{~B}$ study that examined the efficacy of anlotinib in medullary thyroid cancer. Anlotinib is multitarget tyrosine kinase inhibitor targeting the VEGF, PDGF, FGF, and c-kit tyrosine kinase receptors, among others, similar to many of the established TKIs, such as lenvatinib, cabozantinib, sorafenib, and sunitinib. It had previously been tested in smaller trials of medullary thyroid cancer and had shown promise. Disease progression prior to study entry, a critical criterion for studies in medullary thyroid cancer, given the enormous range of aggressiveness in this disease was "required," but no specific time frame was provided. Among 91 patients who were randomly assigned, the progression-free survival (PFS), the study's primary end point, was 20.6 months in the treatment arm versus 11 months in the placebo arm. Comparing these results with published phase 3 studies with vandetanib and cabozantinib, the results from the anlotinib study fall somewhere in between these, which is likely a reflection of the different entry criteria with respect to progression (vandetanib: no progression required, PFS not reached in the treatment arm, 19.3 months in the placebo arm; cabozantinib: progression within 14 months prior to enrollment, PFS 11.4 in the treatment arm versus 4 months in the control arm), rather than true differences in the efficacy of these drugs. The toxicity observed was also similar to that for other anti-VEGF receptor TKIs and included hand-foot syndrome, hypertension, hypertriglyceridemia, and diarrhea. Given a marketplace already crowded with already established similar TKIs, the question is where this drug will find its niche. Perhaps cost and worldwide availability could be its distinguishing feature, but this remains to be seen.

Abstract 6088: Eric Jeffrey Sherman, C. Jillian Tsai, Wanqing Iris Zhi, James Vincent Fetten, Vanessa Wu, Alan Loh Ho, Nadeem Riaz, David G. Pfister, Nancy Y. Lee 2019 Pilot study combining PD-L1 antibody durvalumab with CTLA-4 antibody tremelimumab and stereotactic body radiotherapy (SBRT) to treat metastatic anaplastic thyroid cancer (ATC). J Clin Oncol 37(15 Suppl):6088. abstract. (ClinicalTrials. gov number NCT03122496.) 
THYROID CANCER ASCO 2019 — What's New in Thyroid Oncology? Jochen H. Lorch

Another interesting abstract was presented by Dr. Eric Sherman from Memorial Sloan Kettering Cancer Center and his colleagues. This was a small pilot study of 10 patients with metastatic/incurable anaplastic thyroid cancer. It is well established that the tumor microenvironment of anaplastic thyroid cancer is immunologically "hot," with high numbers of tumor infiltrating lymphocytes (TILs) and abundant macrophages. In this study, the checkpoint inhibitor durvalumab, which targets PD-L1, was combined with tremilimumab, a CTLA-4 antibody. Disappointingly, no response was observed; results from other trials using immunotherapy approaches in anaplastic thyroid cancer, which are currently ongoing at MD Anderson Cancer Center in Houston, TX, and at Dana Farber Cancer Institute in Boston, MA, are eagerly awaited.

Overall, thyroid cancer research was again well represented at ASCO this year. With several interesting studies reaching their enrollment goals in the coming months, there should be a similar number of trials, if not more, to discuss at the annual meeting next year.

This article was prepared or accomplished by the listed author in his personal capacity. The opinions expressed in the article are the author's own and do not reflect the view of the International Thyroid Oncology Group.

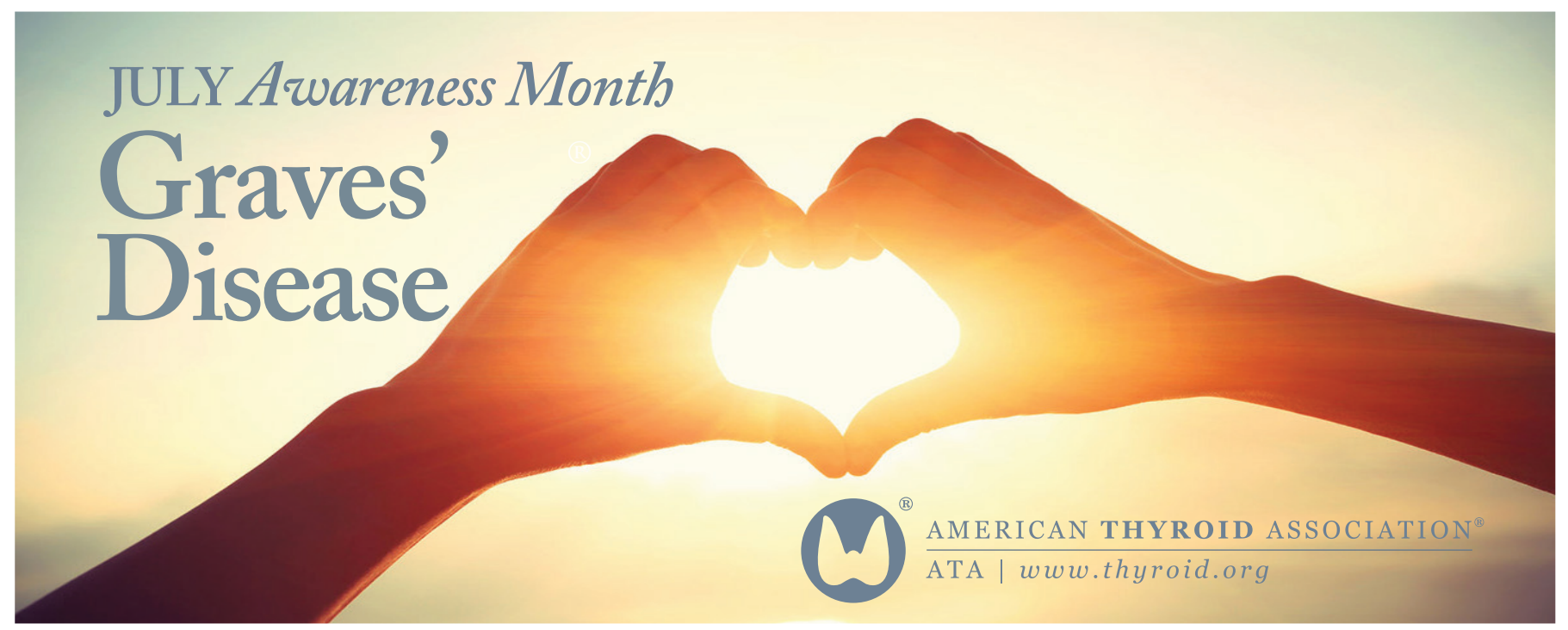

